\title{
Correlative Acoustic Time-Of-Flight Spectroscopy and X-ray Imaging to Investigate Gas-Induced Delamination in Lithium-Ion Pouch Cells during Thermal Runaway
}

\author{
Martin T.M. Pham ', John J. Darst ${ }^{2}$, Donal P. Finegan ${ }^{3}$, James B. Robinson ${ }^{1,4}$, Thomas M.M. \\ Heenan ${ }^{1,4}$, Matt D.R. Kok ${ }^{1,4}$, Francesco Iacoviello ${ }^{1}$, Rhodri Owen ${ }^{1,4}$, William Q. Walker ${ }^{2}$, Oxana V. \\ Magdysyuk ${ }^{5}$, Thomas Connolley ${ }^{5}$, Eric Darcy ${ }^{2}$, Gareth Hinds ${ }^{6}$, Dan J.L. Brett ${ }^{1.4}$, Paul R. Shearing \\ $1,4, *$
}

1. Electrochemical Innovation Lab, Department of Chemical Engineering, University College London, WC1E 7JE, UK

2. NASA Johnson Space Centre, 2101 E NASA Pkwy, Houston, TX 77058, USA

3. National Renewable Energy Laboratory, 15013 Denver W Parkway, Golden, CO 80401, USA

4. The Faraday Institution, Quad One, Harwell Science and Innovation Campus, Didcot, OX11 ORA, UK

5. Diamond Light Source, The Harwell Science and Innovation Campus, Didcot, Oxfordshire, OX11 0DE, UK

6. National Physical Laboratory, Hampton Road, Teddington, London, TW11 0LW, UK

*. Author to whom correspondence should be addressed. Email address: p.shearing@ucl.ac.uk

\begin{abstract}
It remains difficult to detect internal mechanical deformation and gas-induced degradation in lithiumion batteries, especially outside specialized diagnostics laboratories. In this work, we demonstrate that electrochemical acoustic time-of-flight (EA-ToF) spectroscopy can be used as an insightful and fielddeployable diagnostic / prognostic technique to sense the onset of failure. A $210 \mathrm{mAh}$ commercial lithium-ion cell undergoing thermal abuse testing is probed with in situ and operando EA-ToF spectroscopy, together with simultaneous fractional thermal runaway calorimetry (FTRC) and synchrotron X-ray imaging. The combination of X-ray imaging and EA-ToF analysis provides new understanding into the through-plane mechanical deformation in lithium-ion batteries through direct visualisation and the acoustic ToF response. Internal structural changes, such as gas-induced delamination, are identified using EA-ToF spectroscopy due to variations in the attenuation and signal peak shifts. This is corroborated using X-ray imaging, demonstrating EA-ToF spectroscopy as a promising technique for detecting onset of battery failure.
\end{abstract}




\section{Introduction}

Lithium-ion batteries have been the underpinning power supply technology in modern consumer electronics and responsible for the growth of electric vehicles (EVs) in the transport sector. As a result of this, record forecasts for lithium-ion battery production have been chronicled in depth [1]. Moreover, growth predictions are continually revised as efforts to reduce climate impact are made via the development of renewable energy sources and the integration of EVs with a smart electrical grid network [2-4]. Despite their widespread application, concerns regarding the safe deployment of batteries across a range of challenging applications persist.

Lithium-ion batteries can generate significant amounts of heat associated with both electrochemical reactions and ohmic losses. When the rate of heat generation exceeds the rate of heat dissipation, the cell begins to increase in temperature and at a critical temperature, highly exothermic decomposition reactions occur. This process, referred to as thermal runaway, rapidly degrades the anode, cathode, electrolyte, separator and solid electrolyte interphase (SEI) [5-10] and ultimately results in catastrophic failure of the cell. Therefore, understanding the mechanism and onset of thermal runaway is essential for the design of safer lithium-ion batteries. Feng et al. [11] utilised the 'heat-wait-seek' method with extended volume accelerated rate calorimetry to deconstruct the sequence of events during thermally induced failure at increasing temperature. This can be grouped into three significant temperature ranges: at ca. $80^{\circ} \mathrm{C}$, the SEI starts to exothermically decompose. As the anode loses this protective layer, a loss of capacity occurs via de-intercalation of lithium ions from the anode and the anode reacts with the electrolyte producing heat. At ca. $130{ }^{\circ} \mathrm{C}$; the rate of temperature increase slows, and the separator begins to melt (the majority of polyolefin commercial separators consists of polyethylene or polypropylene, which have melting points of ca. $105{ }^{\circ} \mathrm{C}-180{ }^{\circ} \mathrm{C}$ depending on the grade). As the separator melts, this causes micro 'soft' short circuits of the anode and cathode, thereby considerably increasing the rate of reaction, current flow and energy delivered to the regions of short-circuit. These local regions experience greater heating, causing further degradation of the separator. Additionally, less thermally stable cathode materials may begin to decompose at this stage as the temperature continues to increase. At ca. $240^{\circ} \mathrm{C}$, widespread gas generation and decomposition of the cathode, electrolyte and polyvinylidene fluoride binder (PVDF) occurs. These events rapidly produce heat and gas until the build-up of pressure can cause rupture of the cell casing, which is itself a highly destructive and dangerous event [12]. During this sequence of events, it has been reported that up to $2 \mathrm{~L}$ of gas can be generated per amp hour of capacity $[13,14]$.

In this work, thermal abuse fractional thermal runaway calorimetry (FTRC) is used to analyze the thermal evolutions as thermal runaway progresses with electrochemical acoustic time-of-flight (EAToF) probing and co-incident X-ray imaging for internal mechanical changes. FTRC has previously 
been used for cylindrical cell geometry calorimetry to quantify total energy released and to also discern the heat output of ejected and non-ejected material during thermal runaway [11,15-17]. FTRC data has also been used to support statistical assessments which describe the event-to-event variability in thermal runaway responses for a given cell format [15]. Understanding these processes is crucial in both the battery module design and in the development of improved battery management systems (BMS), to minimize excess parasitic mass while ensuring that there is adequate isolation of the characteristic ejection of heat from each cell. From a safety perspective, this second concern is vital; individual cell failure cannot be allowed to trigger cascading thermal runaway throughout an entire module. From a BMS perspective, understanding this process may prevent thermal runaway altogether, by electrically isolating the cell upon detection of signature EA-ToF markers indicative of gas-induced delamination, to prevent further degradation.

Abuse testing methods for lithium-ion batteries typically fall into three broad categories; thermal, mechanical and electrical abuse (external or internal short-circuiting or excessive charge or discharge). The majority of failure testing utilizes the first two [17]. A disadvantage of these techniques is that they are unable to reliably simulate the worst-case scenarios, particularly when all the energy is ejected in a single direction [11,17-20]; furthermore, it is difficult to determine exactly when the thermal runaway will initiate, especially in situations of thermal abuse. Other abuse methods, such as oven tests or accelerating rate calorimetry, have limitations in the time taken to achieve the critical temperature to initiate thermal runaway, compared to FTRC which was used in this work. These insufficiently fast failure tests may cause the electrolyte to evaporate due to initial venting, thus resulting in a dry electrode assembly, possibly yielding inaccurate thermal failure insights [11]. Surface temperatures in excess of $600{ }^{\circ} \mathrm{C}$ are common during failures, which can occur within a few seconds $[16,17,19]$. Therefore, in batteries, the ability to forecast and understand ruptures and failures will provide key insight into module safety and performance [17].

In-depth insight with direct non-destructive imaging during thermal runaway has been demonstrated by Finegan et al. [5,16,19] and Robinson et al. [21] with synchrotron X-ray and thermal imaging, respectively. Electrochemical and structural changes have also been investigated by several authors [22-25] using techniques such as neutron imaging and electrochemical impedance spectroscopy (EIS). EIS has been used by Fernandez et al. [26] to quantify the degradation mechanisms (conductivity loss, loss of active material and loss of lithium inventory) within a BMS; however, limitations in accuracy exist which are dependent upon the measurements and models [27]. Moreover, EIS measurements are affected by environmental and operating conditions, including temperature, C-rate, state-of-charge (SoC), depth-of-discharge (DoD) and cabling configuration. Furthermore, models can require complex computation for a limited operating range of conditions. 
EA-ToF spectroscopy is applicable when materials are not stressed in tension or compression beyond their elastic limit. In this work, an ultrasonic wave is used to apply a force into component materials within a pouch cell within their elastic limit, ensuring a repeatable and valid characteristic spectroscopy. The relationship between the speed of sound $(c)$ of the ultrasonic wave used in EA-ToF spectroscopy, is dependent upon elastic modulus $(E)$ and density $(\rho)$, as defined by the Newton-Laplace equation (Equation 1).

$$
c=\sqrt{\frac{E}{\rho}}
$$

The difference in time-of-flight (ToF) between interfaces is also dependent on the thickness of each layer, with a thicker layer producing a proportionately delayed ToF. Hsieh et al. [28] have previously discussed the importance of material density on acoustic wave propagation in electrode assembly materials. Robinson et al. [29] inferred changes in the ToF, and thereby the location of the electrode from the transducer as a result of changes in the acoustic signal at the same SoC. Further EA-ToF work conducted by Robinson et al. [30] characterised behaviours induced during cycling of lithium-ion pouch cells at a range of C-rates, such as potential stresses in the electrode. In addition to ToF shifts, another important change in EA-ToF spectroscopy is the attenuation in acoustic intensity. When the ultrasonic signal is transmitted through each layer of material, the wave is scattered, reflected and absorbed to different extents. The acoustic impedance of a system, $\Delta Z$, as a function of the change in density, $\Delta \rho$, and the elastic modulus, $E$, is given by:

$$
\Delta Z=\sqrt{|\Delta \rho| E}
$$

(Equation 2)

Gas exhibits significantly higher attenuation than liquids or solids as it is very poor at transmitting ultrasonic signals. This is shown as a reduction in acoustic intensity or loss of reflected signal during EA-ToF analysis. Galushkin et al. [31,32] investigated the generation of gases within an electrode assembly during cycling including their contributions to thermal runaway, these gases and phases changes within an electrode assembly would be identifiable with acoustic spectroscopy. As the lack of propagation of the ultrasonic wave pulsed by the transducer and the change in density at the interfaces of different phases would exhibit high attenuation and delay in ToF.

Hsieh et al. [28] have demonstrated that EA-ToF has the capability to determine SoC and state-ofhealth $(\mathrm{SoH})$ of pouch cells at low C-rates $(0.2 \mathrm{C}, 0.1 \mathrm{C}, 0.05 \mathrm{C})$, given prior acoustic measurements and voltage correlation to deduce relative ToF shift. Models developed by Davies et al. [33] displayed robustness in predicting SoC to $1 \%$ accuracy for both intact and damaged cells. Additional models by 
Davies et al. [33] evaluated SoH by comparison of EA-ToF measurements at full charge after 20 cycles, also displayed similar accuracy. Spatially resolved acoustic ToF characterisation by Robinson et al. [29] has shown promise in probing the internal structure as a function of SoC. Other acoustic techniques using guided waves of an order of magnitude lower in frequency have probed the $\mathrm{SoC}$ and $\mathrm{SoH}$ of cells [34,35]. Ladpli et al. [35] used algorithms to decompose the acoustic waveforms into simpler constituents to analyse the acoustic-electrochemistry phenomena in order to predict SoC and SoH. Gold et al. [34] have also utilised ultrasonic waves at $200 \mathrm{kHz}$ to resolve SoC determination over one cycle. The use of the lower frequency signals facilitated the characterisation of a graphite electrode by analysis of arrival times of the slower, compressional waves at different SoC. Previous work conducted by the authors [30] has demonstrated temporally resolved EA-ToF spectroscopy as a correlative proxy for determining the effects of high-rate cycling and identifying electrochemical stiffness effects in batteries. However, there remain uncertainties regarding the mechanisms which cause structural degradation; an indication of the onset of failure, which have not to date been characterised by EA-ToF analysis.

In this paper, electrode delamination and gas formation in lithium-ion batteries is investigated using coupled EA-ToF spectroscopy and X-ray imaging. These events are known causes of cell degradation and precursors to the onset of thermal runaway. Gas-induced delamination in a planar electrode assembly is prominently visible via EA-ToF spectroscopy due to the significant changes in the measured attenuation and shift in the waveform peak location. Laboratory and synchrotron X-ray imaging with X-ray CT are used to highlight the internal phenomena which occur during defect driven gas-induced delamination and thermal abuse FTRC and to provide correlative validation. The paper is split into two parts, the first demonstrates the application of laboratory X-ray imaging with EA-ToF spectroscopy to identify gas formation in a cell cycling under normal conditions; the second utilises EA-ToF spectroscopy with high-speed synchrotron imaging to observe and characterise thermal runaway.

This is the first time to the authors' knowledge where acoustic spectroscopy and X-ray imaging have been effectively combined to explore the performance and failure of $\mathrm{Li}$-ion batteries. The techniques used in concert provide unique insight into the gas induced delamination and are subsequently used to explore the nucleation and propagation of failure. Understanding, and improving the safety of Li-ion batteries remains a critical challenge, and therefore we anticipate this work will have a significant impact across the battery community; providing new fundamental insight into the failure process, and a platform of new capabilities for future studies of thermal runaway behaviour. 


\section{Experimental}

\section{Acoustic ToF integrated calorimetric set-up}

Acoustic spectroscopy analysis using ultrasonic testing is based on propagation and reflection of sound waves through materials. When applied to electrochemical power systems the approach is generally referred to as EA-ToF spectroscopy, and was first demonstrated for battery applications by Steingart $e t$ al. $[28,33]$. In this work, ultrasonic waves are transmitted perpendicular to the electrode layers. Figure 1 shows how the ultrasonic signal reflects off each layer which provides the ToF difference, $\Delta t$ or the characteristic response. These reflections which are picked up by the transducer give rise to the waveform signal peaks shown in Figure $1 b$. 
(a)

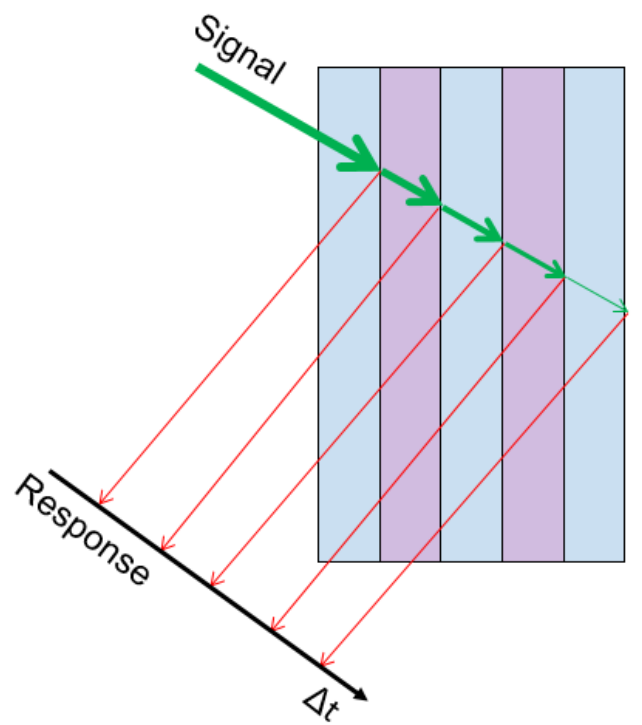

(b)

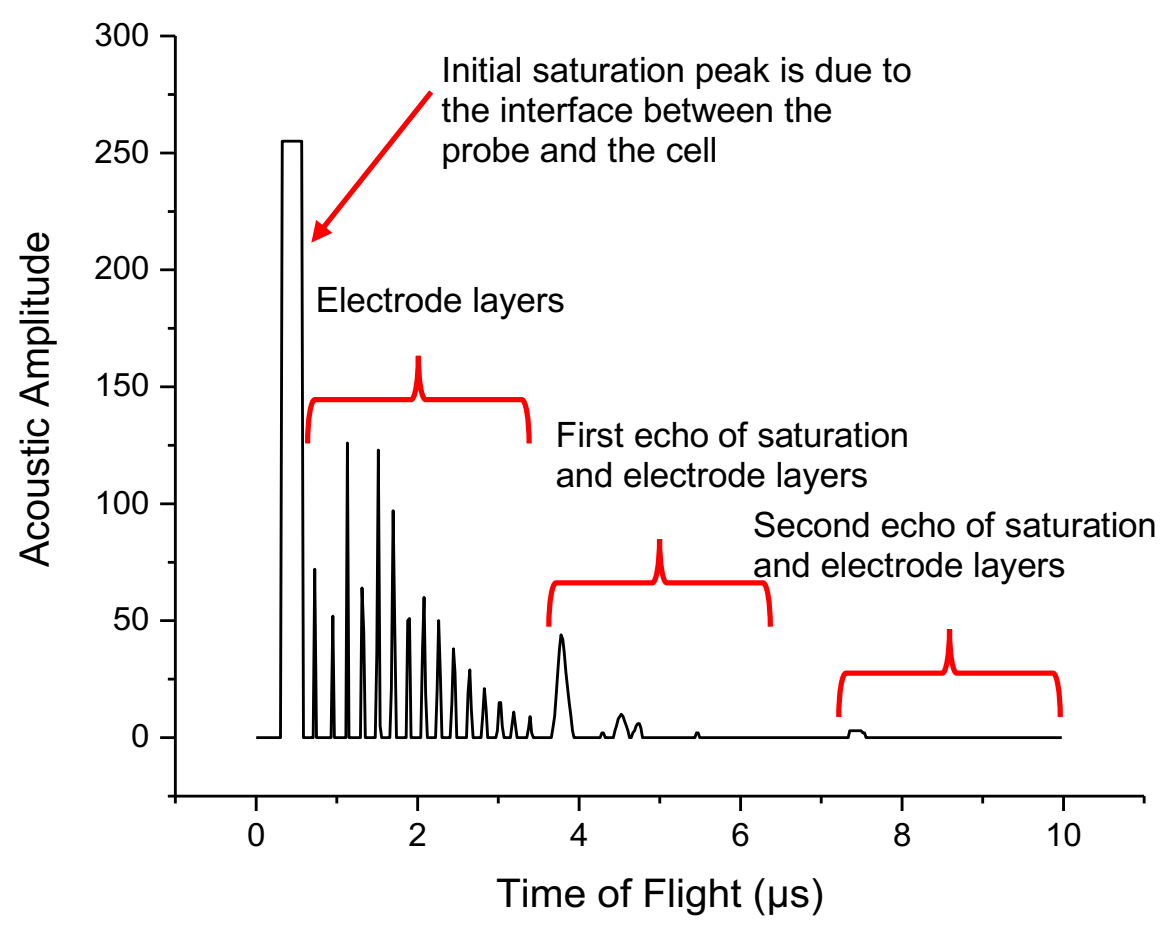

Figure 1: (a) As the ultrasonic pulse propagates through each layer, each interface produces an observed reflection, with insight into evolution of structural properties that correspond to SoC and SoH, given by the attenuation and observed ToF shift. An ultrasonic pulse which is at an angle to the electrode layers has been used to clearly show the ToF difference in the reflected wave, which is the response sensed by the transducer. (b) The initial waveform at the start of the experiment from Figure 3, this data set is a static acoustic ToF response with continuous acquisition providing the ToF shift analysis. EA-ToF data is plotted as a spectrogram for timeresolved analysis with the correlative $X$-ray imaging and X-ray $C T$.

All acoustic ultrasound ToF measurements were obtained via an Olympus Epoch 650 ultrasonic flaw detector (Olympus Corp, Japan) with a pulse-echo contact transducer (M110-RM, Olympus Corp, 
Japan). The $6 \mathrm{~mm}$ piezoelectric transducer element is capable of frequencies up to $5 \mathrm{MHz}$, with the chosen frequency of $2.25 \mathrm{MHz}$ used for all measurements. Couplant suitable for high temperature applications (H-2, Olympus Corp, Japan) was applied to maintain interfacial contact between the cell and transducer to facilitate propagation of the ultrasound wave into the cell. Constant pressure was applied behind the transducer during simultaneous X-ray imaging. Operando laboratory X-ray imaging was conducted with a $200 \mathrm{~g}$ weight placed on top of the sensor. During synchrotron fractional thermal runaway calorimetry, a compressive force of ca. $27 \mathrm{~kg}\left(82.74 \mathrm{~N} \cdot \mathrm{cm}^{-2}\right.$ measured by a torque wrench, correlating torque to compression) was applied. The experimental set-ups are shown in Figure 2.

(a)

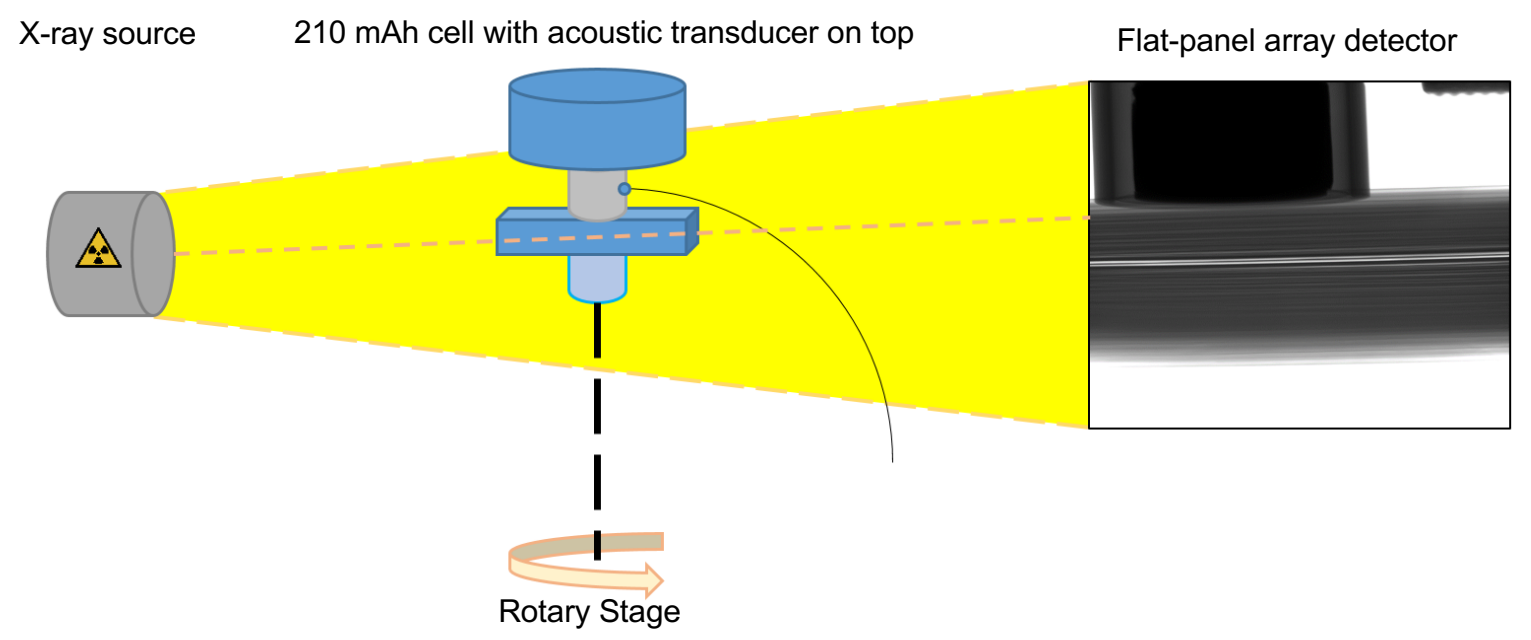

(b)

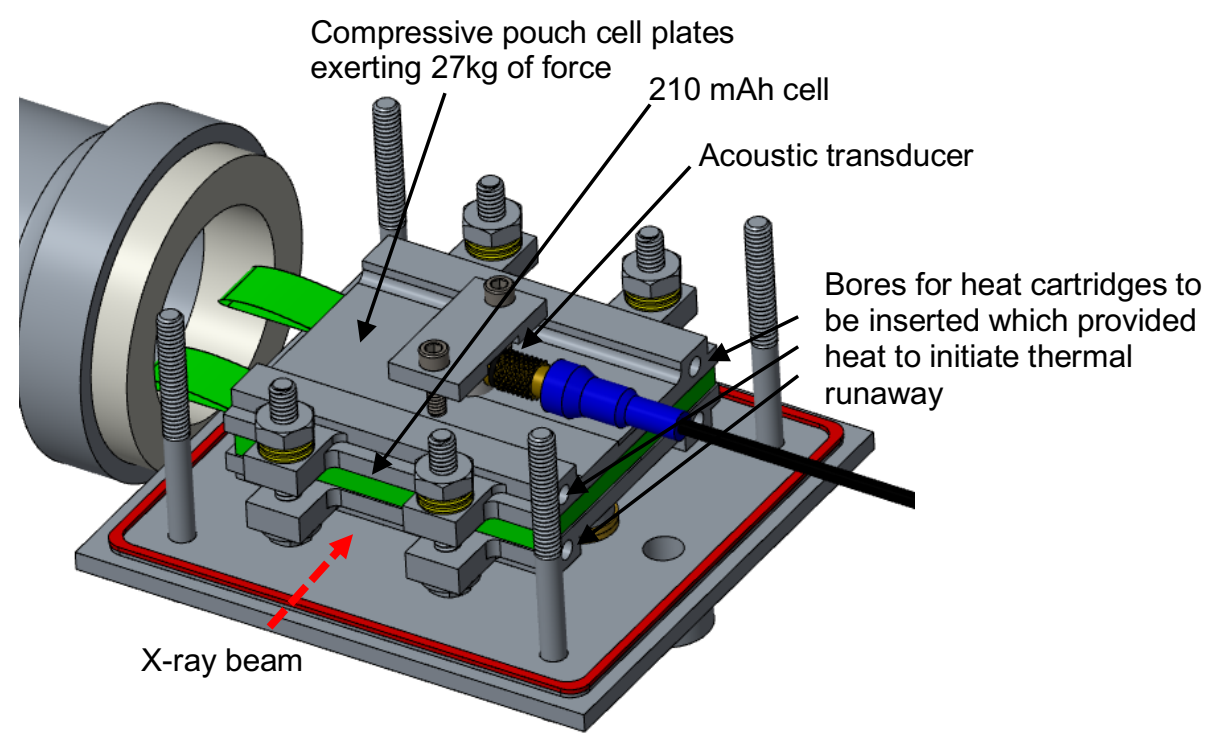

Figure 2: (a) A simple schematic of the experimental set-up used in the Nikon XT H225 to produce simultaneous acoustic spectroscopy and X-ray imaging. (b) $3 D$ rendering of the ultrasonic transducer in the pouch cell calorimeter cell chamber stack under compression. 
The magnitude of the measured reflected ultrasonic signal by the transducer is determined by the gain magnification, which was set at $60 \mathrm{~dB}$. The voltage applied to the piezoelectric transducer was $200 \mathrm{~V}$. The resolution of each waveform data sample, with 495 discrete mapping points equally spaced across the range of $10 \mu \mathrm{s}$, provides a ToF resolution of $20.2 \mathrm{~ns}$ for each data point. The ultrasound waveform data was transferred from the Epoch 650 through an RS232 connection and output files acquired by an open-source Python code described in previous work by Steingart et al. [28,33,36].

A pouch cell calorimeter was designed to accommodate thermal, mechanical and electrochemical abuse for a range of pouch cell dimensions (up to $50 \mathrm{~mm} \times 50 \mathrm{~mm}$ ). The temperature was measured at the surface of the cell with type-K thermocouples. The experimental set-up [Figure 2(b)] served multiple purposes; the bores seen in Figure 2(b) were fitted with heater cartridges which provided heat to initiate failure. The compressive plates containing the heater cartridges ensured more effective thermal contact between the heated plates and the surface area of the cell through the duration of the test. This also counteracted any insulation effect of gas generation which may reduce thermal conductivity between the heater cartridges and the cell. This facilitated reliable initiation of thermal runaway as the active materials within the cell could be reliably heated until failure occurred as swelling due to gas production did not occur at the cell-compression plate interface. This compression also ensured that EA-ToF probing was possible throughout the duration of the test as gas accumulation did not occur through the acoustic probe plane, which caused loss of signal shown in Part I, where no compression of the cell was used.

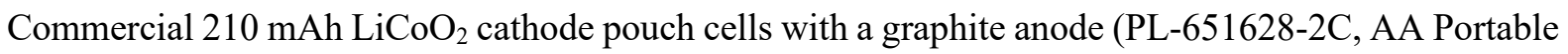
Power Corp., CA, USA) were charged to $4.2 \mathrm{~V}$ using a constant current, constant voltage (CCCV) protocol. The constant current charge was performed at $0.5 \mathrm{C}$ with the voltage then held constant at 4.2 $\mathrm{V}$ until the current dropped below $0.05 \mathrm{C}$. The cell was then left at open circuit potential for $20 \mathrm{~min}$ before being put into compression plates for failure testing. During calorimetry testing, failure was induced at different heat fluxes to provide varied insight into the processes of thermal abuse leading to thermal runaway, which may have different time constants. Heating was applied using heater cartridges inserted into the compressive plates seen in Figure 2b. Heating was switched off immediately after the onset of widespread thermal runaway which was observed using X-ray radiography.

Electrochemical testing was achieved using a Gamry Interface 1010E potentiostat (Gamry Instruments, Pennsylvania, USA). During defect identification cycling, the cell CCCV cycling was conducted at 0.5 $\mathrm{C}$ constant current, from ca. $3.6 \mathrm{~V}$ to $4.2 \mathrm{~V}$, then using a constant voltage protocol until the current cutoff of 0.1 C. Discharge cut-off was $2.75 \mathrm{~V}$, with a discharge current of $0.5 \mathrm{C}$. 


\section{$\mathrm{X}$-ray radiographic imaging:}

Laboratory-based X-ray radiographic imaging was conducted using a Nikon XT H225 (Nikon Metrology, UK), with a $2 \mathrm{~K} \times 2 \mathrm{~K}$ PerkinElmer 1620 Detector; this was performed simultaneously to the EA-ToF measurements. The imaging parameters were chosen to ensure appropriate $X$-ray penetration of the cell as well as sufficient contrast to show gas-induced delamination and other structural changes. An accelerating voltage of $130 \mathrm{kV}$ and a current of $70 \mu \mathrm{A}$ were used. No filter was used and one frame per second (fps) radiograph acquisition was achieved with a pixel resolution of $5.55 \mu \mathrm{m}$.

High-speed X-ray radiography at $20 \mathrm{fps}$ and $580 \mu$ s exposure time was performed at the I12 beamline [37] at Diamond Light Source (DLS), the radiography was used to capture the transient internal architecture throughout testing, whilst simultaneously capturing EA-ToF data. The cells were imaged under a $74 \mathrm{keV}$ monochromatic parallel beam with a pixel size of $16.26 \mu \mathrm{m}$ and a field-of-view (FOV) of $20 \mathrm{~mm} \times 12 \mathrm{~mm}$ (Horizontal $\times$ Vertical). A $\mathrm{Gd}_{3} \mathrm{Ga}_{5} \mathrm{O}_{12}(\mathrm{GGG})$ scintillator and Miro 310 detector were used for all high-speed imaging experiments.

Selected frames highlighting the significant structural evolution during gas formation and thermal runaway from these radiographic videos are shown in this paper, with the videos illustrating failure provided in the Supplementary Information.

\section{X-ray micro-CT:}

Non-destructive X-ray Computed Tomography (CT) was conducted using two systems, a Nikon XT H 225 (Nikon Metrology, UK) and a Zeiss Xradia 520 Versa (Carl Zeiss, USA). Both CT systems utilise a tungsten target. The accelerating voltages and beam energy were selected based on the attenuation of the samples and the contrast of materials. Radiographs were obtained at incremental angles while rotating the vertical axis in the middle of the region of interest. These acquired radiographs were subsequently reconstructed using commercial software packages (CT Pro 3D and Zeiss XMReconstructor for Nikon and Zeiss CT systems respectively), which use algorithms based on the standard filtered back-projection or the Feldkamp-David-Kress (FDK) method. Collection parameters are described in Table 1. 
Table 1: Imaging configurations for X-ray CT.

\begin{tabular}{|c|c|c|c|c|c|c|c|}
\hline System & Voxel Size & FOV & $\begin{array}{c}\text { Exposure } \\
\text { Time }\end{array}$ & $\begin{array}{c}\text { Accelerating } \\
\text { voltage }\end{array}$ & Power & Filter & Projections \\
\hline $\begin{array}{c}\text { Nikon XT } \\
\text { H } 225\end{array}$ & $24.5 \mu \mathrm{m}$ & $\begin{array}{c}40 \times 40 \\
\mathrm{~mm}\end{array}$ & $1 \mathrm{~s}$ & $180 \mathrm{kV}$ & $18.5 \mathrm{~W}$ & $1 \mathrm{~mm}$ & 3176 \\
\hline $\begin{array}{c}\text { Zeiss Xradia } \\
520 \text { Versa } \\
(0.4 \times\end{array}$ & $7.7 \mu \mathrm{m}$ & $10 \times 10$ & $25 \mathrm{~s}$ & $160 \mathrm{kV}$ & $10 \mathrm{~W}$ & N/A & 3201 \\
\hline $\begin{array}{c}\text { magnification) } \\
\text { mm }\end{array}$ & & & & & & \\
\hline
\end{tabular}

Post collection segmentation and visualisation of the reconstructed tomograms were processed using Avizo Fire 9.5 software (Thermo Fisher Scientific, France). 


\section{Results and Discussion}

\section{Laboratory Imaging}

A static cone-beam tungsten source was used for the laboratory X-ray imaging; as a result, layers that were horizontally co-incident or parallel to the centre of this beam were much brighter in the radiograph due to the lack of flat-field corrections applied to radiography. In each of the frames seen in Figure 3, the brighter horizontal layer co-incident with the centre of the X-ray beam was used as a reference point due to the stationary source and a fixed sample holder below the cell. As the cell was discharged at 0.5 $\mathrm{C}$, a greater proportion of the cell above this reference point was observed and therefore it can be observed that the pouch cell experiences mechanical expansion, which is corroborated by a reduced proportion of the transducer in the FOV. At $162 \mathrm{~min}$, delamination of the electrode layers under the sensor and gas generation can be observed and correlated with the loss of ultrasonic signal. Correlating EA-ToF spectroscopy are seen in Figure 5.

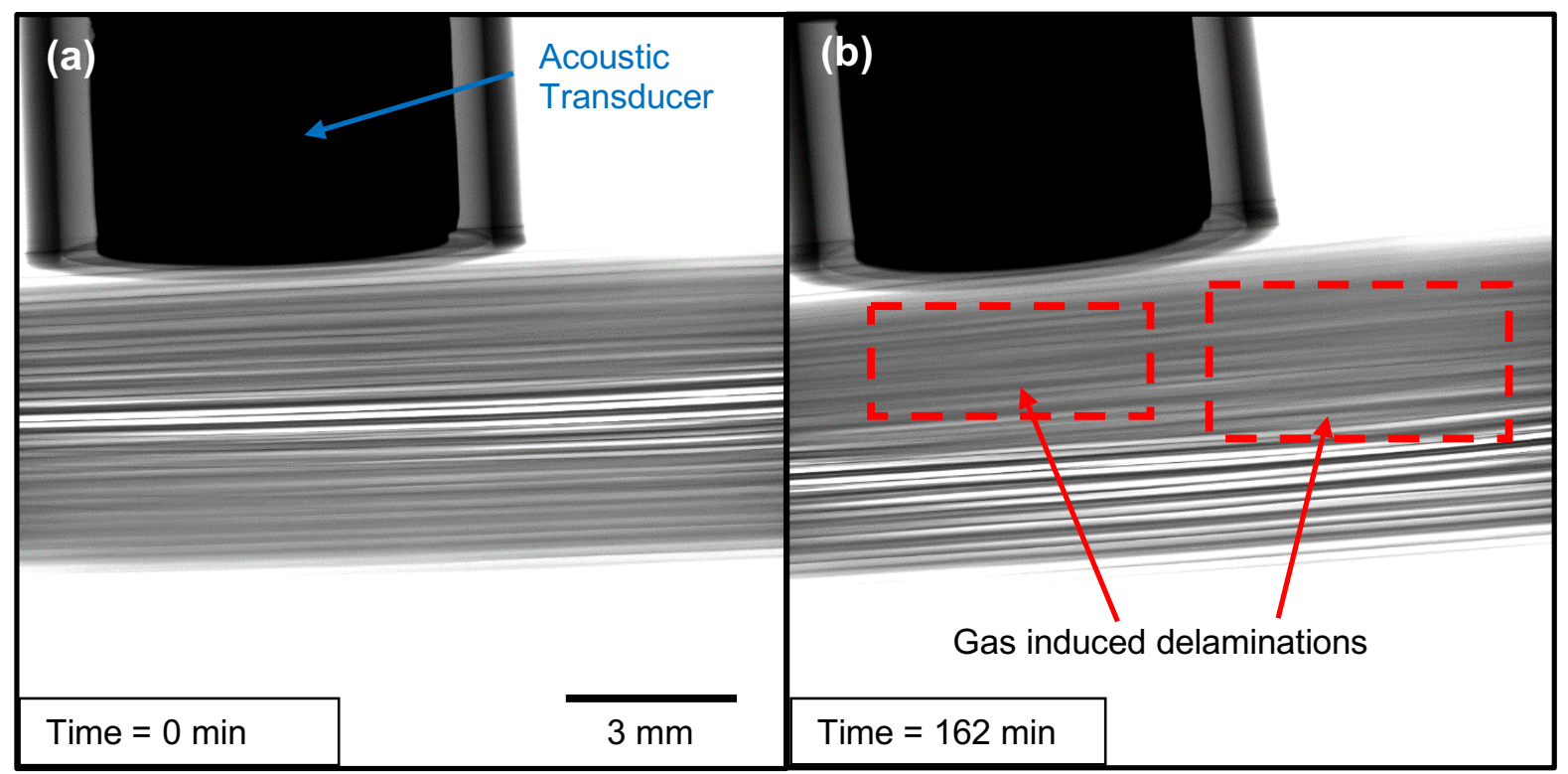

Figure 3: Radiographs depicting the expansion of the pouch cell layers, with the horizontal bright-line being the centre of cone-beam with no incident angle. The radiography video can be seen in Supplementary material. (a) Initial radiograph from which relative comparisons were made. (b) Gas-induced delamination during discharge observed.

The acoustic spectrogram shown in Figure 4 represents the individual EA-ToF snapshot data acquired throughout the duration of the experiment with the amplitude of the waveform depicted by the colourmap key. Given the nature of the acoustic response signals, the first horizontal white line (located at ca. $0.625 \mu \mathrm{s}-1.25 \mu$ s seen in Figure 4(a)) describes the initial saturation of the transducer which does not change with time. The subsequent red lines provide information pertaining to the first electrode and 
subsequent material interfaces until a ToF of ca. $7.5 \mu \mathrm{s}$, where the sensed ultrasonic wave reflections are echoed.

(a)

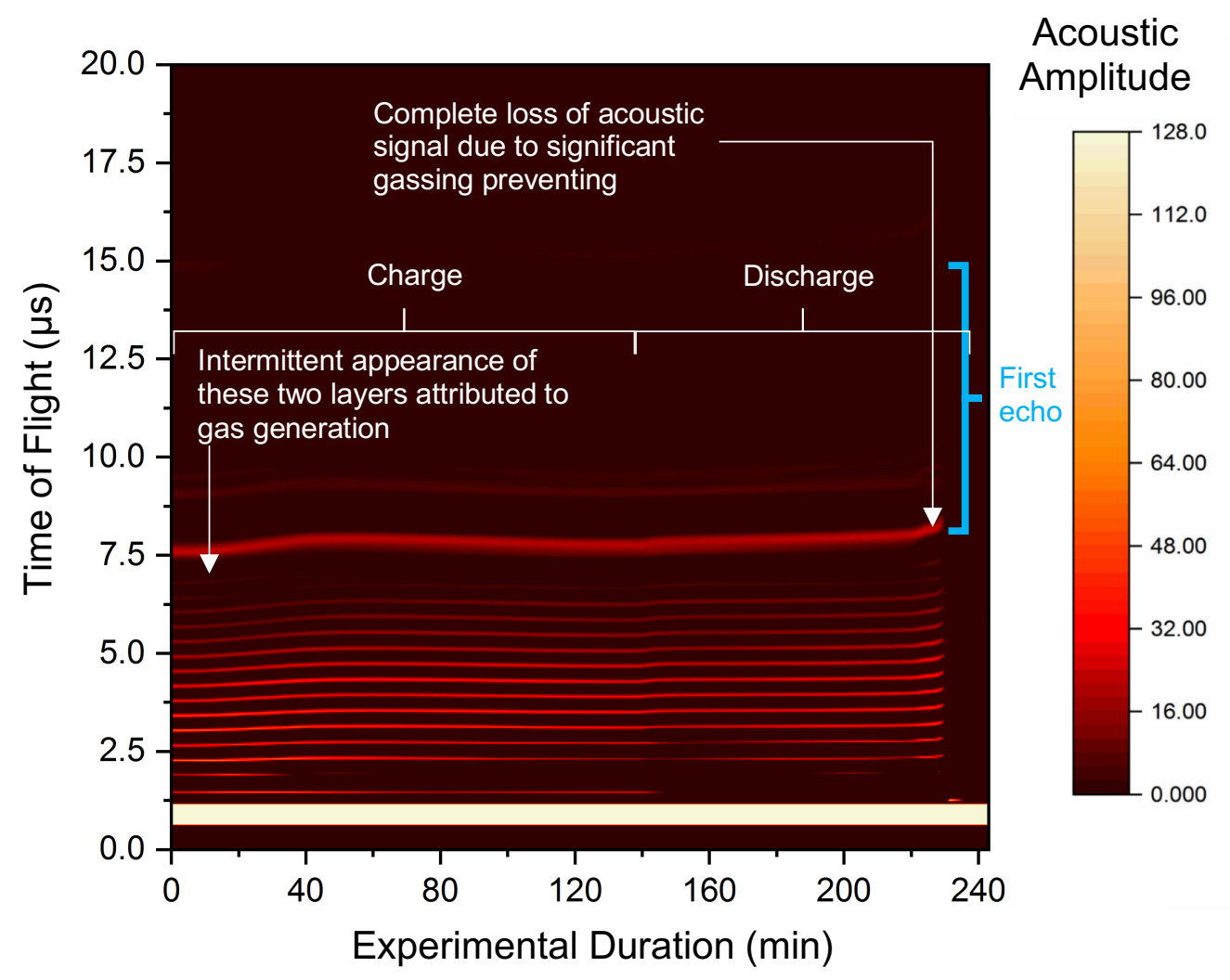

(b)

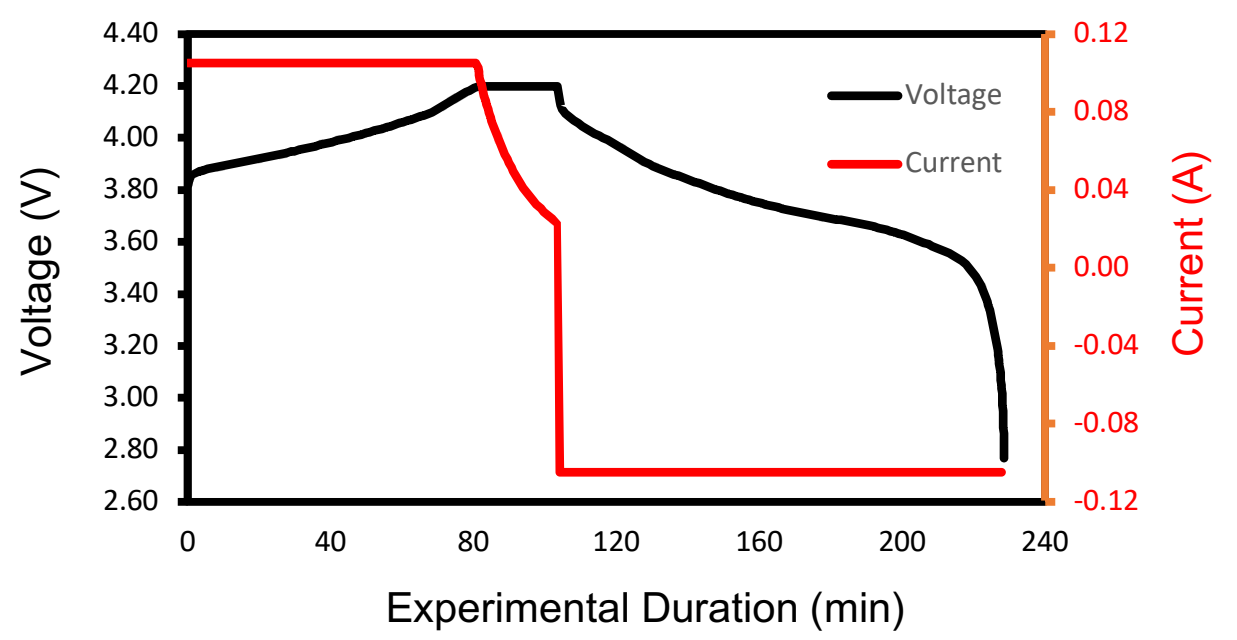

Figure 4: Acoustic ToF spectrogram plotted with respect to experimental duration which can be correlated to voltage or SOC, the characteristic wave amplitude is shown by the colour scheme. Shifts in ToF through the battery were observed; with significant changes at ca. $40 \mathrm{~min}, 45 \mathrm{~min}$ and $230 \mathrm{~min}$. The highly reflected first wave consistent throughout all ultrasonic measurements and observed at ca. $1.3 \mu \mathrm{s}$. At ca. $7.5 \mu \mathrm{s}$, the first echo of the reflected signals appears, with a fainter second echo at ca. $14.9 \mu \mathrm{s}$. The acoustic amplitude is displayed in arbitrary units. 
Previous work [30] with this commercial $210 \mathrm{mAh}$ cell has seen consistent and reliable operation when cycled within the specified operating parameters of voltage, current and temperature. ToF shifts and attenuation associated with density changes due to lithiation and delithiation are immediately observable and have been explained previously [30]. However, in this experiment, the cell was cycled with a constant force applied behind the transducer. Gas formation and mechanical delamination was observed in both X-ray imaging and EA-ToF spectroscopy.

Initial indicators of gas generation were observed with EA-ToF spectroscopy [Figure 4], as the cell was cycled. An intermittent appearance of the internal electrode layers at ca. $6.25 \mu$ s ToF can be observed during the duration of the experiment. The final two layers of the electrode assembly, observed with EA-ToF spectroscopy disappeared and reappeared. This can be ascribed to gas generation and then gas movement away from the EA-ToF probed region because of the additional force behind the transducer. This gradual accumulation and movement of gas reduced the acoustic amplitude, which is reflected in the diminished intensity of the colour in Figure 5.

A further indication of the formation of gas was the fluctuation in the intensity of the waveform at ca. $140 \mathrm{~min}$, represented by the acoustic amplitude (measured in arbitrary units, AU) - which decreased from approximately 96 to below 32, as highlighted in Figure 5. At the start of the experiment, at ca. $6.25 \mu \mathrm{s} \mathrm{ToF}$, there was a clear reduction in the acoustic intensity (ca. $24 \mathrm{AU}$ ). This intensity fluctuated throughout the experiment; the signal was lost intermittently and gradually declined in acoustic intensity (ca. $12 \mathrm{AU}$ at $140 \mathrm{~min}$ ). This intensity drop was also observed at the two EA-ToF reflected peaks adjacent to the $2.5 \mu \mathrm{s}$ ToF (seen as red adjacent lines either side of the $2.5 \mu$ s ToF marker).

At 230 min, a second more apparent feature is observed in Figure 4, after a rapid increase in ToF, loss of signal was observed. This coincided with decreased acoustic intensity of reflections of the layers close to the acoustic sensor beforehand (at $2.5 \mu \mathrm{s}$ ToF and from $150 \mathrm{~min}$ to $230 \mathrm{~min}$ ). This can be attributed to the formation of gas during cycling. This was a key indicator in cell failure, as previous literature has shown the onset of gassing within the cell predicates actual thermal runaway and ejection of material $[19,38]$. There were portions of the transducer which could successfully transmit and sensed the reflected signals and some which did not. This lack of homogeneity in the gas, liquid and solid phases with the electrode assembly was responsible for the presence of acoustically visible electrode layers further away but not those adjacent across the surface area of the transducer as the transducer averaged the reflected signal sensed across its surface area.

The region of interest where acoustic ToF measurements were taken [shown inset in Figure 5], was observed and shown as an orthogonal slice from the reconstructed tomogram of the electrode layers before degradation and after gas generation. At the start of the experiment, the uniformity of the 
electrode layers at the centre of the cell is evidenced by the inset cross-sectional orthoslice [Figure 5(a)], which was also reflected in the acoustic data [Figure 5]. 

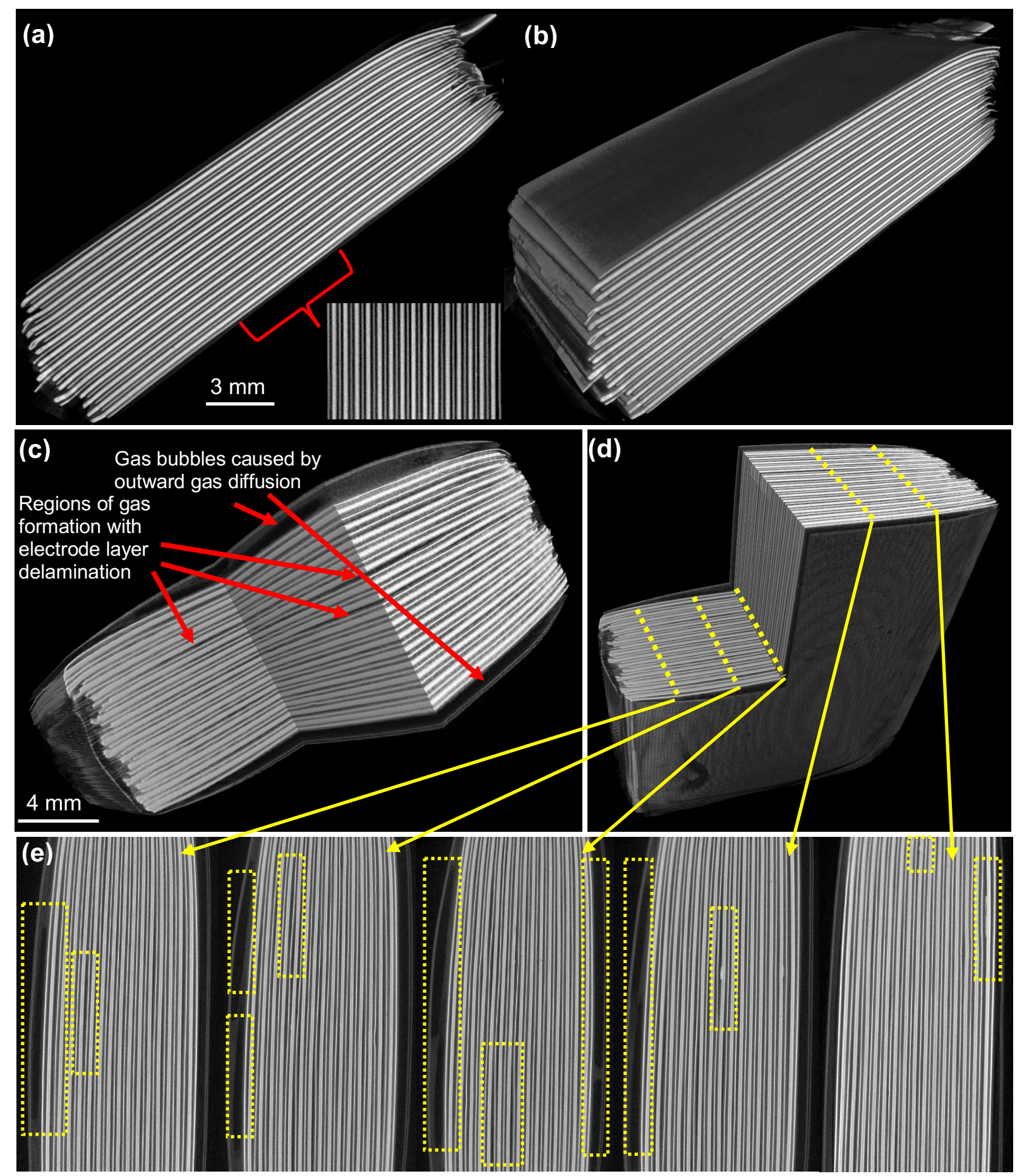

Figure 5: (a) An X-ray orthoslice cross-section of the cell prior to cycling and failure, the EA-ToF probed region is shown inset. (b) An X-ray CT of the $210 \mathrm{mAh}$ lithium-ion pouch cell before the detection of defect or failure. (c) Post-mortem X-ray CT of the region of interest, which was probed via EA-ToF spectroscopy during cycling. The highly attenuating gas pocket at the edge of the pouch cell, outside the electrode assembly and within the outer casing, is responsible for the loss in the acoustic signal. This provided visualisation via CT after gas-induced delamination with regions of interest depicted. (d). Side profile of the acoustic region of interest, with the location of the orthoslice provided. (e). Orthoslices highlighting some of the mechanical defects and features visible via acoustic ToF. Scale bar used in (c) applicable to (d) and (e). XCT acquisition parameters can be found in Table 1. 
Figure 5 highlights multiple instances of electrode delamination as well as accumulation of the gases produced at the surface of the electrode assembly in contact with the polymer cell casing after cycling was completed. Comparison of X-ray CT before [Figure 5a and Figure 5b] and after failure [Figure 5c, Figure 5d and Figure 5e], shows the increasing delamination of the electrode, with the defects and gas regions circled in Figure 5. Additional comparison between the acoustic and X-ray imaging data, highlighted that these regions were nucleation sites for gas formation. Throughout the duration of the experiment, the trend of fluctuating acoustic amplitude throughout the 'deepest' EA-ToF observable electrode layers, can be attributed to gas formation and later movement away from the EA-ToF probed region. This was due to the $200 \mathrm{~g}$ weight applied to the transducer which slowly forced the gas laterally, away from the probe. Eventually, gas generation and diffusion of gas through the electrode layer resulted in macroscopic gas accumulation between the electrode layer and the cell casing. 


\section{Correlative Synchrotron Imaging}

To further evaluate the value of EA-ToF spectroscopy to characterise thermal runaway, co-incident high speed X-ray imaging and EA-ToF probing was conducted at a synchrotron facility during thermal abuse testing of two identical cells to those used in the laboratory tests described above.

Radiography captured the failure process of both Cell 1 and Cell 2 [Figure 6(a) and Figure 6(b) respectively] in the two thermal runaway tests conducted. This provided insight into correlating acoustic signatures shown in EA-ToF spectroscopy with internal phenomena seen by X-ray imaging and postmortem CT. Due to the experimental set-up in a synchrotron experimental hutch, the acquisition of the EA-ToF data commenced at experimental time $=0 \mathrm{~s}$; however, X-ray imaging started later due to safety protocols to ensure no personnel were present in the experimental hutch. Thus, the onset of thermal abuse via the heater cartridges occurred several min (ca. 6 min $20 \mathrm{~s}$ and ca. $4 \mathrm{~min} 30 \mathrm{~s}$ for Cell 1 and Cell 2 respectively) into the experiments when both EA-ToF probing and X-ray imaging data could both be acquired simultaneously. For Cell 1, heating started at $6 \mathrm{~min} 20 \mathrm{~s}$ in the first thermal runaway experiment seen in Figure 6(a-i). The structural changes seen in Figure 6(a-ii), recorded at $20 \mathrm{fps}$, and were correlated with the ultrasonic ToF reflections sensed by the transducer in Figure 7(a). After 6 min $35 \mathrm{~s}$, shown in the Figure 6(a-ii), mechanical deformation of the layers adjacent to the transducer was observed, which preceded local gas formation. The shadow which is seen to appear adjacent to the face of the transducer [Figure 6(a-ii)] indicates the disintegration of the laminar electrode format, thus less distinct and high contrast edges are seen in Figure 6(a-i). 


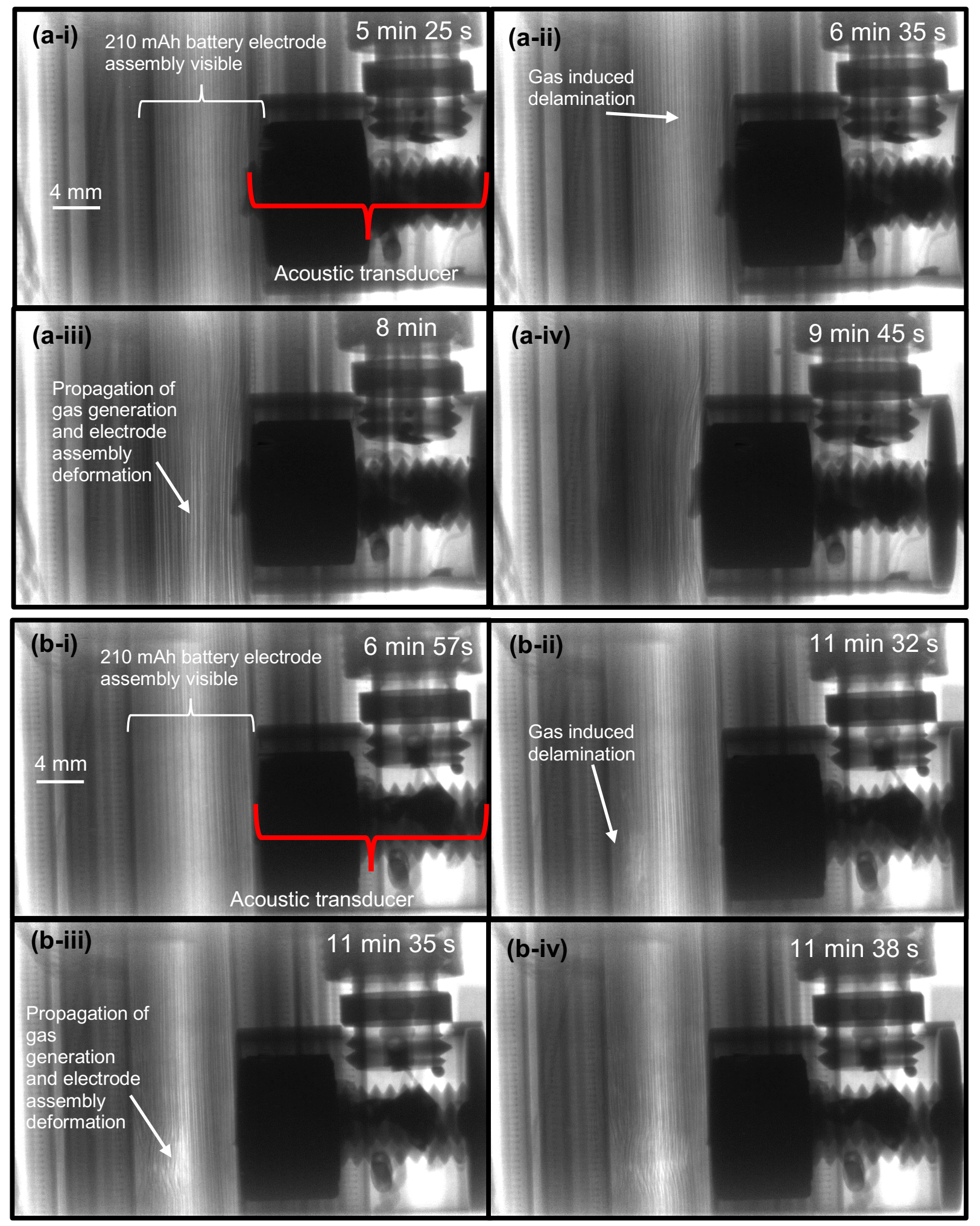

Figure 6: (a) Selected radiography frames from Cell 1 highlighting significant structural changes during thermal runaway in the first failure test. The pouch cell calorimeter was at an angle to the X-ray beam path during this first test, hence the visible screw next to the transducer and the left-hand side of the cell was covered by some of the aluminium compression plate. Delamination and gas generation predicated the initiation of widespread thermal runaway. Thermal runaway, the ejection of gas and material and the subsequent recompression of the cell are shown in the four frames. (a-i) Heating has been applied for a minute and minor delamination was 
observed, this radiograph can be used as a reference for significant changes induced due to thermal abuse. (a-ii) After 2 min $10 \mathrm{~s}$ of heating, the first major signs of delamination can be observed. (a-iii) Widespread gas generation and thermal runaway occurred. (a-iv) Thermal runaway and gas generation ended, the compressive pouch cell chamber stack has forced the electrode layers together. (b) The radiography of Cell 2 during the second thermal abuse test conducted at a higher heat flux in the aluminium plates of the $210 \mathrm{mAh}$ commercial lithiumion cell. (b-i) At 6 min 57 s, the cell has expanded with increasing temperatures as heating had been applied for ca. $30 \mathrm{~s}$. (b-ii) Formation of the gas phase was visible. (b-iii) Onset of widespread thermal runaway caused further gas generation. (b-iv) Widespread thermal runaway and gas generation which led to pressure build-up and eventually ruptured the cell casing.

The onset of widespread thermal runaway of Cell 1 during the first experiment was seen in the third radiograph frame [Figure 6(a-iii)]. Through the X-ray visible plane, the entire electrode assembly exhibited delamination. Thermal decomposition of the $\mathrm{LiCoO}_{2}$ cathode, electrolyte and other active material was seen as fracture of the electrode assembly with rapid gas generation. The gas-induced delamination observed throughout the cell, caused the pressure to peak as observed by the maximum swelling of the cell. The increased pressure due to gas generation, coupled with the force of the compressive plates resulted in a breach of the external casing of the cell. These dynamic structural evolutions were are apparent in Supplementary Movie 2.

After $9 \min 45 \mathrm{~s}$, thermal runaway had concluded, with no generation and ejection of gas and no ejection of material. The compressive plates applied continual force to the transducer to ensure perpendicular movement of gas to the ultrasonic imaging plane and allowed the layers to be acoustically propagative as well as preserving the intimate contact of the cell-transducer interface. Thermal runaway caused the electrode to compress in a deformed manner. This reduced distance between electrode layers was correlated and seen in Figure 7(a) after $10 \mathrm{~min}$, as the reduced distance between electrode layers has yielded a greater acoustically visible depth and higher acoustic amplitude.

For Cell 2, in the second thermal runaway experiment seen in Figure 6(b), heating started at ca. 4 min $20 \mathrm{~s}$ [Figure 6(b)]. The electrode rupture can be observed in Figure 6(b-ii) from the gradual formation and accumulation of the gas from exothermic decomposition of the materials, which was not as severe as previously seen in Figure 6(a). This rupture occurred in the EA-ToF probed region and therefore was reflected in a slower progression in the delay of the ToF shift in Figure 7. In Figure 6(b-ii), the initial formation of gas visible in the X-ray and acoustic FOV correlated with the acoustic ToF shift at 11 min $32 \mathrm{~s}$.

Despite the greater heat flux applied to Cell 2, a less violent failure was observed; as shown by the predominantly intact electrode assembly with a gaseous void at the end of the experiment. This is due 
to the greater temperature gradient from the heater to the entire battery and the temperature gradient across the battery, as a result of the greater heat flux. The high heat flux resulted in a higher local cell temperature adjacent to the cartridge heater, which caused local thermal runaway but did not allow enough time for the entire cell's contents to reach the critical temperature.

Given the compressive force provided by the experimental set-up [Figure 2(b)], the ultrasound wave propagated further through the pouch cell, therefore, more layers of the electrode assembly were identified without saturation of the transducer's reflection sensor or increasing the sensor's FOV. In addition, the initial four layers experienced a greater acoustic amplitude and a reduced ToF between the characteristic waveform peaks seen in Figure 7(a) and 7(b). This can be explained by the compressive force applied, which reduced the acoustic amplitude losses between interfaces. Consequently, this improved propagation of the ultrasonic signal as there was improved interfacial contact between layers. 
(a)

Acoustic

Amplitude

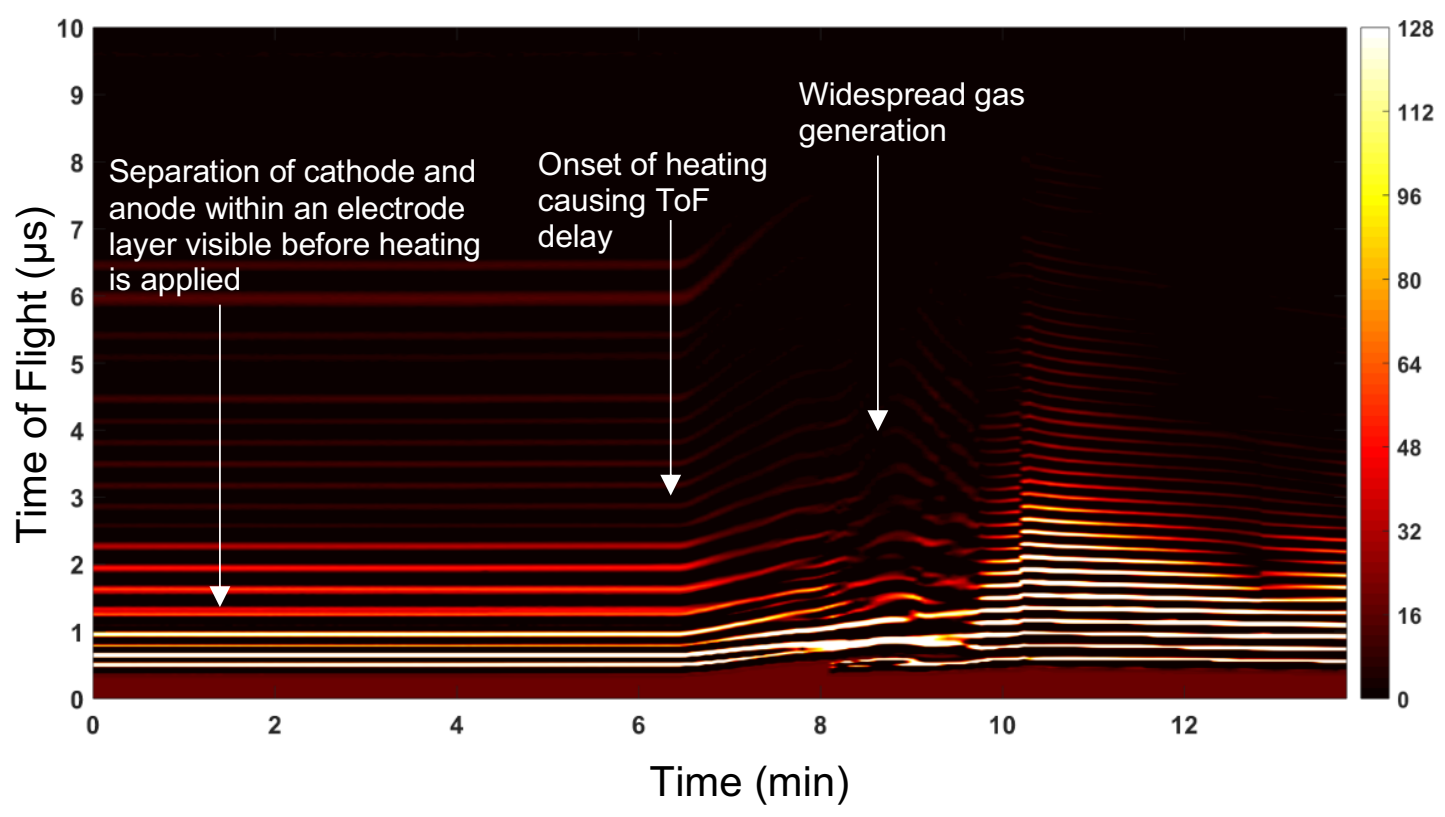

(b)

Acoustic

Amplitude

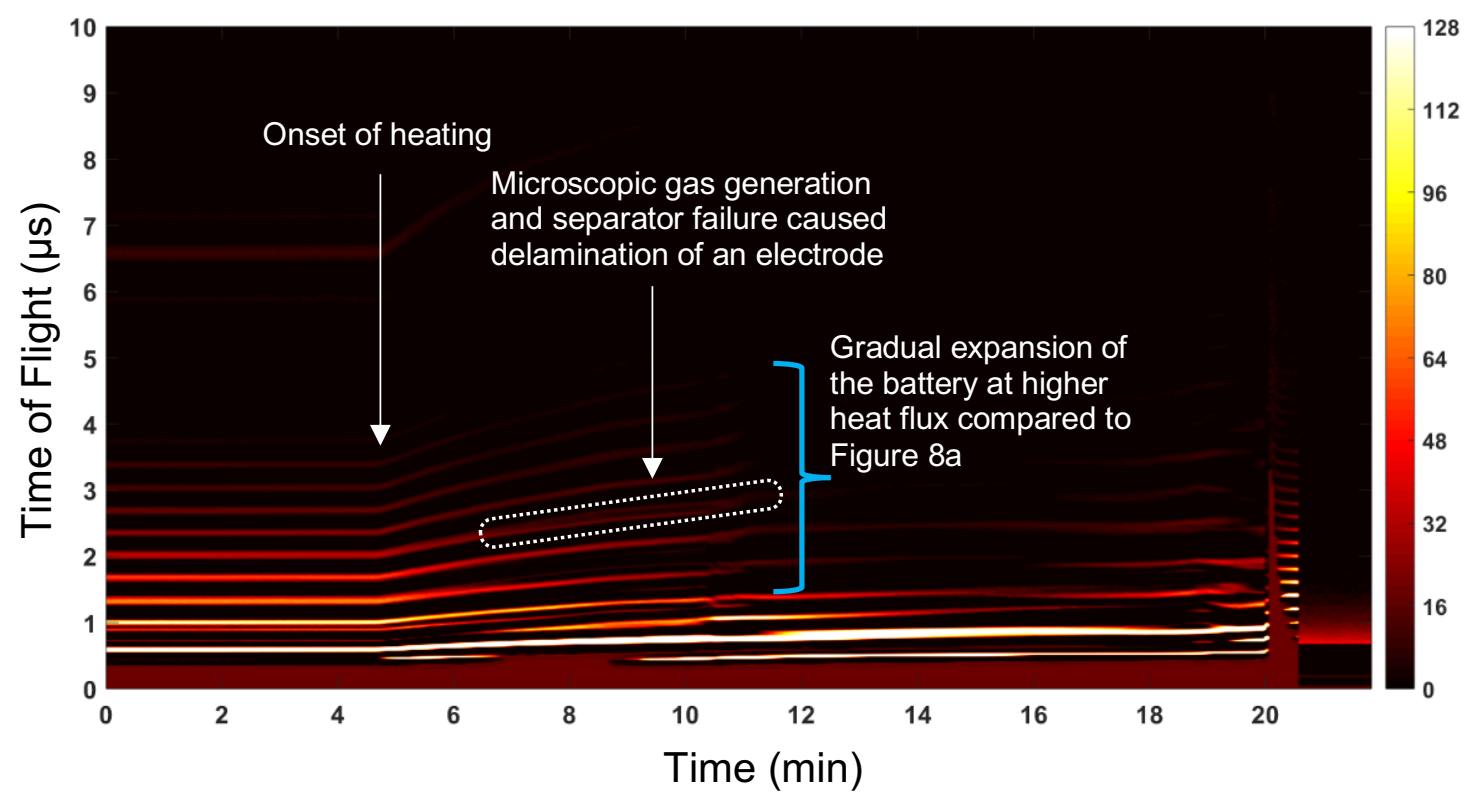

Figure 7: (a) EA-ToF spectrogram of Cell 1 during the first failure test, with highlighted regions of interest during induced thermal runaway of a $210 \mathrm{mAh}$ commercial pouch cell. This spectrogram corresponded to the radiography shown in Figure 6(a). Onset of heating at approximately 6 min $20 \mathrm{~s}$ into the test. (b) EA-ToF spectrogram of Cell 2 during the second failure test, with highlighted regions of interest during thermal runaway of a $210 \mathrm{mAh}$ commercial pouch cell in the second test, corresponded to the radiography shown in Figure 6(b). Onset of thermal runaway at approximately 4 mins 20 s into the test with the main features of interest identified on the AS plot. The acoustic amplitude is displayed in arbitrary units. 
During the gradual thermal abuse of Cell 1, the onset of heating is clear ca. 6 min $20 \mathrm{~s}$ into the test as shown in Figure 7(a), as there is a distinct trend of delayed ToF of the ultrasonic wave due to cumulative propagation through the previous layers. This was attributed to decreasing density with increasing temperature and therefore poorer propagation via molecules, which are further apart at greater temperatures due to greater kinetic energy. Consequently, this reduced the ultrasonic wave velocity through the solid electrodes which caused delays in propagation and reflection of the ultrasonic signal therefore a trend of delayed ToF during heating before thermal runaway. Microscopic gas formation and delamination of the electrode which causes expansion or spatially divergent electrode material was visible during radiography at every layer of the pouch cell seen in Figure 6(a-iv) with the layers seen by EA-ToF spectroscopy, contorted away from the transducer on the right-hand side. The onset of widespread macroscopic gas generation is labelled at $8 \mathrm{~min}$ whereby widespread gas generation and rupture of the cell occurred. This gas-induced delamination contributed to the reduction in the ultrasonic wave velocity through the cell, which was exhibited in the ToF for each subsequent electrode layer, thus the cumulative effect of this delay was more apparent for the layers at greater ToF. As the cell progresses through thermal runaway despite the removal of heat at ca. $8 \mathrm{~min}$, the significant gas volume which had formed internally away from the transducer, did not propagate the ultrasound wave and thus no reflection of the attenuated waves is observable despite the very high compressive force. Gas formation begins to occur throughout the cell and once the gas phase reaches a significant volume, each subsequently reflected wave declines in acoustic amplitude with a general trend of delayed shift in ToF. At later experimental duration, these reflected waves no longer appear as the gas propagation progresses. After ca. $8 \mathrm{~min}$, the significant volume of gas has caused maximum expansion of the pouch cell, whereby the internal pressure causes the pouch cell to burst. The constant compressive force applied by the pouch cell chamber coupled with the lack of internal pressure after the cell ruptured and gas ejected, allowed visibility of electrode layers further away from the transducer. The highly compressed pouch cell, which has ruptured and ejected gas, seen in Figure 7(a) after ca. 10 min, as thermal runaway has passed and thermal decomposition to produce gas has stopped.

Initiation of failure of Cell 2 during the second thermal runaway test can be identified with heating induced and observed at ca. $4 \mathrm{~min} 30 \mathrm{~s}$, as a result of thermal decomposition of the electrolyte and density changes of the active material. Consequently, this exhibited a characteristically similar trend and change in waveform as shown previously in Figure 7(a). It is suspected that due to the higher heating rate applied to Cell Two, the heat was not uniformly distributed to the active material within the cell hence the longer duration of widespread thermal runaway and a gradual mechanical expansion of the electrode assembly. Once the onset of failure had occurred, thermal abuse was stopped and the process of failure was extended compared to the first abuse test. More gradual heating applied to Cell 1 during the first failure test ensured a lower temperature gradient across the cell and between the cell and the heat cartridges, thus all the active material within Cell 1 was at a higher average temperature 
than Cell 2. This higher temperature gradient between the heater cartridges and the cell and across the active material of Cell 2 was reflected in a slower propagation of thermal runaway and a less catastrophic failure probed by the acoustic transducer. This was observed via a more gradual ToF shift seen in Figure 7(b). This highlighted the difference in rate of loss of acoustic signal - which was seen to a much greater extent in Figure 7(a). However, as a consequence of the slower thermal runaway dynamics of Cell 2, the gas generation lasted longer and there was a longer time during which reflected ultrasonic signals were absent (ca. 11 min to 16.5 mins).

For Cell 2, an interesting phenomenon was observed at the electrode layer initially situated at $2.05 \mu \mathrm{s}$ ToF, which began to split around 6 min. During the onset of thermal runaway, gas was observed to be generated between the anode and cathode with radiography; this is mirrored by the splitting of the single peak reflected wave shown and circled in Figure 7(b). The greater retention of the electrode structural integrity seen in Figure 6(b), which was reflected in acoustic measurements with a less sudden shift in ToF of the electrode layers adjacent to the cell, visible at low ToF. The progressive disappearance of reflected ultrasonic waveforms from a later ToF to an earlier ToF is suspected to be due to layers further away being hidden by macroscopic gas formation during thermal runaway and later rupture of the cell which caused widespread loss of EA-ToF visible electrode layers.

It is difficult to quantitatively evaluate the degree to which ToF and attenuation have been affected by the gas phase. During thermal runaway, the dynamic fluidisation of the electrode assembly, especially with the transient nature of the composition of this gas-phase at different phases of thermal runaway rapidly changes due to the different extent of reactions and number of reactions that can occur. Later accumulation of the gas phase due to macroscopic gas generation and movement to the external surface of the electrode assembly but within the aluminium casing of the cell, formed a gas layer which attenuated the ultrasonic wave significantly. Consequently as loss of acoustic signal was observed in Figure 4 at ca. $230 \mathrm{~min}$. This physical phenomenon was also observed in Figure 7 before the ejection of gas and material in thermal runaway before recompression due to the plates of the pouch cell calorimeter chamber. 


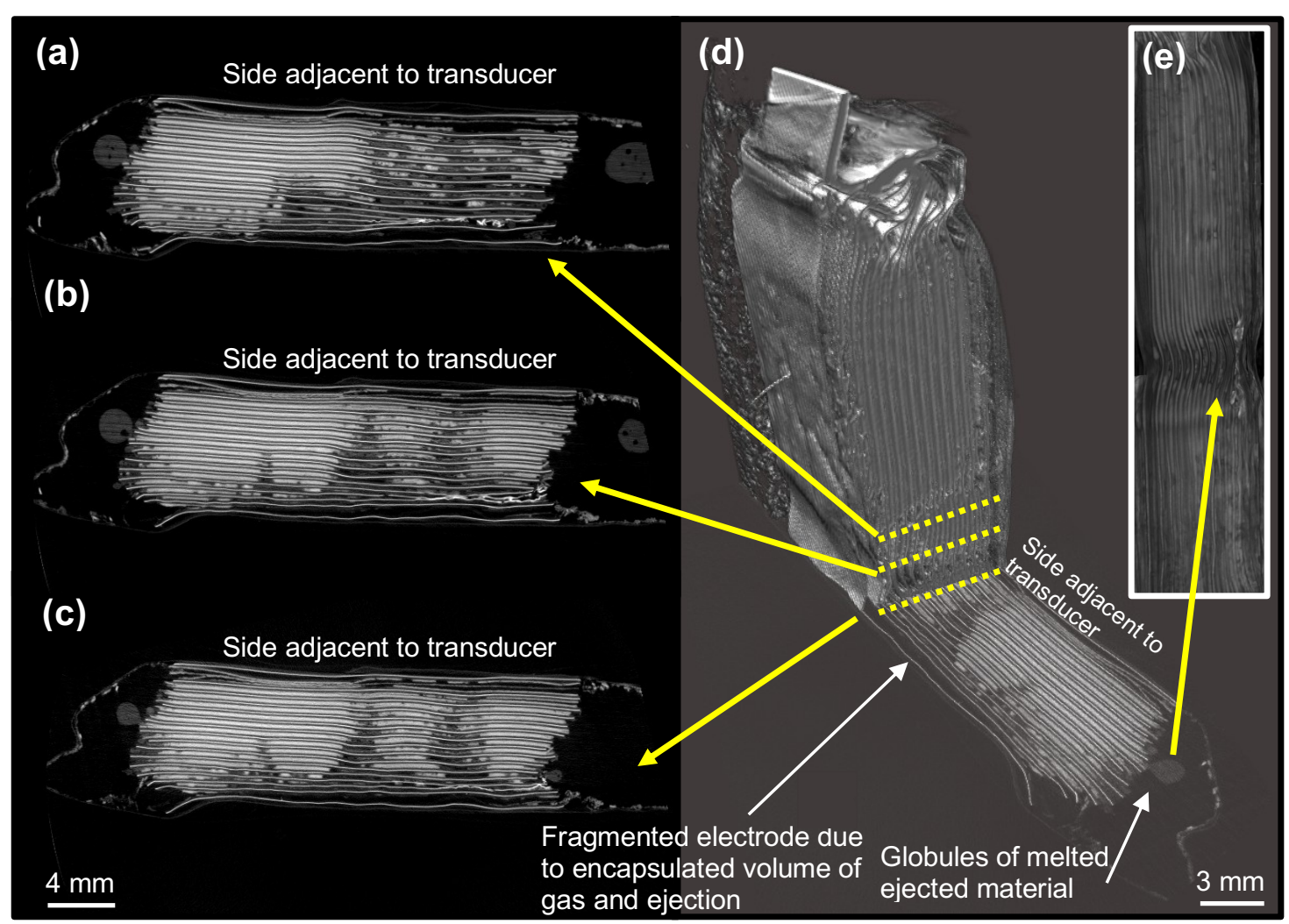

Figure 8: Radiography and tomography of Cell 2 after thermal runaway where: (a) top, (b) middle, (c) bottom are the corresponding orthogonal slices illustrating the gas formation regions in line with the ultrasonic transducer. This EA-ToF probed region is ca. $6 \mathrm{~mm}$ in diameter. (d) An internal and external view of the cell with the side profile view of the damage caused by pathway formed for gas and material ejection shown inset (e).

The remains of Cell 1 were insufficiently intact for post-mortem CT. Thus, only the tomogram of Cell 2 from the second thermal runaway experiment cell is seen in Figure 8. The internal pouch cell architecture was assessed with post-mortem tomography, for which the Nikon CT imaging configurations can be found in Table 1. Reconstruction and visualisation with Avizo 9.5 software, exposed the mechanical damage during thermal runaway. There were some internal un-combusted remains of the cell electrode assembly which have remained intact, primarily the highly attenuating cathode and copper current collector, which were visible on the acoustic transducer side of the cell and on the left side in the radiographs. The damaged electrode layers and ejection of material was stark on the highly attenuating cathode layers, with the two furthest layers from the transducer undergoing complete delamination and structural collapse.

The encapsulated gas volume observed due to the loss of material by ejection seen in Figure 8(d), reinforced the loss of acoustic signal at later ToF. Ejected material was seen on the external far side of the cell with some globules of previously melted material visible. The fragmented structure supported the hypothesis of loss of reflected ultrasound signal depth due to a lack of transmission through the gas gap seen in Figure 8. 
The EA-ToF probing analysed in Figure 7 was correlated with time to the X-ray imaging, which produced radiographs shown in Figure 6. The expansion of the electrode layers and attenuation of the acoustic signal was observed in the radiography seen in Figure 7. The expansion of the electrode layers during cycling, gas-induced delamination and gas formation throughout the three experiments detailed here were consistent with the ToF delay in the acoustic spectrogram and X-ray imaging.

\section{Conclusions}

In-situ and operando EA-ToF spectroscopy has been demonstrated to be a robust tool in identifying gas formation and probing mechanical deformation within cells, which has been coupled with X-ray radiography and CT to provide a comprehensive tool for cell failure analysis. Through-plane, temporally-resolved structural changes contributing to the onset of degradation and failure have been identified using EA-ToF spectroscopy. These sequences of events causing mechanical failure have been linked to degradation in electrochemical performance, capacity retention and thermal response. In-situ $\mathrm{X}$-ray imaging and ex-situ X-ray CT confirmed these observations, which were also corroborated with the principles of EA-ToF spectroscopy.

Due to the propagative properties of ultrasound through different phases such as attenuation and wave velocity, integration into battery management systems would allow direct measurement and analysis of the onset of gassing within complex modules; as well as $\mathrm{SoC}, \mathrm{SoH}$ and electrochemical performance. Gas formation and delamination are prime indicators of impending thermal runaway, which could be prevented with acoustic analysis implemented in the BMS, with a spatially resolved array of transducers offering potential for prognostic, temporally resolved, in-situ and operando ultrasonic-based analysis. However, limitations arise in precisely quantifying the attenuation and ToF shifts compared to the theoretically expected values.

EA-ToF spectroscopy has potential as a powerful, non-destructive and field-accessible technique which would see applications in EV battery modules on-board or in-line metrology during battery manufacture or evaluation for second-life/recycling. 


\section{Acknowledgements}

The authors would like to acknowledge the expertise provided by Zoran Bilc during the calorimeter design, assembly and instrumentation. Special thanks to the supporting facilities and technical staff at the NASA JSC Energy Systems and Test Area (ESTA). We are grateful for the provision of synchrotron beamtime provided by Diamond Light Source on beamline I12 and for allowing us to use their facilities (Experiment EE20903-1). The authors would like to acknowledge the EPSRC for supporting the energy storage work in the Electrochemical Innovation Lab (EP/R020973/1; EP/R023581/1; EP/N032888/1; EP/N001583/1. EP/P009050/1; EP/M009394/1; EP/M014371/1). This work was partly funded by the National Measurement System of the UK Department of Business, Energy and Industrial Strategy. The authors would also like to acknowledge the Royal Academy of Engineering for funding Robinson and Shearing through ICRF1718\1\34 and CiET1718 respectively and the Faraday Institution (EP/R042012/1 and EP/R042063/1). The authors also acknowledge the STFC for supporting Shearing and Brett (ST/K00171X/1). The authors would like to acknowledge Quentin Meyer and Daniel Steingart for their assistance in sourcing the equipment and supplying the basic code used to obtain the data. This work was authored in part by the National Renewable Energy Laboratory, operated by Alliance for Sustainable Energy, LLC, for the U.S. Department of Energy (DOE) under Contract No. DE-AC36-08GO28308. Funding was provided by the U.S. Department of Energy Office of Energy Efficiency and Renewable Energy Vehicle Technologies Office. The views expressed in the article do not necessarily represent the views of the DOE or the U.S. Government. The U.S. Government retains and the publisher, by accepting the article for publication, acknowledges that the U.S. Government retains a nonexclusive, paid-up, irrevocable, worldwide license to publish or reproduce the published form of this work, or allow others to do so, for U.S. Government purposes. 


\section{References}

[1] European Commission. Report on Raw Materials for Battery Applications. Brussels: 2018.

[2] Mwasilu F, Justo JJ, Kim EK, Do TD, Jung JW. Electric vehicles and smart grid interaction: A review on vehicle to grid and renewable energy sources integration. Renew Sustain Energy Rev 2014. https://doi.org/10.1016/j.rser.2014.03.031.

[3] European Environment Agency. Electric vehicles from life cycle and circular economy perspectives - TERM 2018. Report 13/2018. 2018. https://doi.org/10.2800/77428.

[4] Su W, Eichi H, Zeng W, Chow MY. A survey on the electrification of transportation in a smart grid environment. IEEE Trans Ind Informatics 2012. https://doi.org/10.1109/TII.2011.2172454.

[5] Finegan DP, Scheel M, Robinson JB, Tjaden B, Di Michiel M, Hinds G, et al. Investigating lithium-ion battery materials during overcharge-induced thermal runaway: An operando and multi-scale X-ray CT study. Phys Chem Chem Phys 2016;18:30912-9. https://doi.org/10.1039/c6cp04251a.

[6] Chen Z, Qin Y, Ren Y, Lu W, Orendorff C, Roth EP, et al. Multi-scale study of thermal stability of lithiated graphite. Energy Environ Sci 2011;4:4023-30. https://doi.org/10.1039/c1 ee01786a.

[7] Ren D, Liu X, Feng X, Lu L, Ouyang M, Li J, et al. Model-based thermal runaway prediction of lithium-ion batteries from kinetics analysis of cell components. Appl Energy 2018;228:63344. https://doi.org/10.1016/j.apenergy.2018.06.126.

[8] Lopez CF, Jeevarajan JA, Mukherjee PP. Experimental Analysis of Thermal Runaway and Propagation in Lithium-Ion Battery Modules. J Electrochem Soc 2015;162:A1905-15. https://doi.org/10.1149/2.0921509jes.

[9] Fu Y, Lu S, Li K, Liu C, Cheng X, Zhang H. An experimental study on burning behaviors of 18650 lithium ion batteries using a cone calorimeter. J Power Sources 2015. https://doi.org/10.1016/j.jpowsour.2014.09.039.

[10] Wang Q, Ping P, Zhao X, Chu G, Sun J, Chen C. Thermal runaway caused fire and explosion of lithium ion battery. J Power Sources 2012. https://doi.org/10.1016/j.jpowsour.2012.02.038.

[11] Feng X, Fang M, He X, Ouyang M, Lu L, Wang H, et al. Thermal runaway features of large format prismatic lithium ion battery using extended volume accelerating rate calorimetry. $\mathrm{J}$ Power Sources 2014;255:294-301. https://doi.org/10.1016/j.jpowsour.2014.01.005.

[12] Finegan DP, Darcy E, Keyser M, Tjaden B, Heenan TMM, Jervis R, et al. Identifying the Cause of Rupture of Li-Ion Batteries during Thermal Runaway. Adv Sci 2018. https://doi.org/10.1002/advs.201700369.

[13] Orendorff CJ, Lamb J, Nagasubramanian G, Fenton KR, Langendorf JL. Through Materials 
Development Lithium - Ion Battery Safety Challenges 2015.

[14] Golubkov AW, Fuchs D, Wagner J, Wiltsche H, Stangl C, Fauler G, et al. Thermal-runaway experiments on consumer Li-ion batteries with metal-oxide and olivin-type cathodes. RSC Adv 2014;4:3633-42. https://doi.org/10.1039/c3ra45748f.

[15] Walker WQ, Darst JJ, Finegan DP, Bayles GA, Johnson KL, Darcy E, et al. Decoupling of heat generated from ejected and non-ejected contents of 18650-format lithium-ion cells using statistical methods. J Power Sources 2019;415:207-18. https://doi.org/10.1016/j.jpowsour.2018.10.099.

[16] Finegan DP, Darcy E, Keyser M, Tjaden B, Heenan TMM, Jervis R, et al. Characterising thermal runaway within lithium-ion cells by inducing and monitoring internal short circuits. Energy Environ Sci 2017;10:1377-88. https://doi.org/10.1039/c7ee00385d.

[17] Finegan DP, Darst JJ, Walker WQ, M. M. Heenan T, Hack J, Thomas JC, et al. Modelling and experiments to identify high-risk failure scenarios for testing the safety of lithium-ion cells. J Power Sources 2019;417:29-41.

[18] Lamb J, Orendorff CJ. Evaluation of mechanical abuse techniques in lithium ion batteries. J Power Sources 2014. https://doi.org/10.1016/j.jpowsour.2013.08.066.

[19] Finegan D, Scheel M, Robinson JB, Tjaden B, Hunt I, Mason TJ, et al. In-operando highspeed tomography of lithium-ion batteries during thermal runaway. Nat Commun 2015;6:110. https://doi.org/10.1038/ncomms7924.

[20] Finegan DP, Tjaden B, M. M. Heenan T, Jervis R, Michiel M Di, Rack A, et al. Tracking Internal Temperature and Structural Dynamics during Nail Penetration of Lithium-Ion Cells. J Electrochem Soc 2017. https://doi.org/10.1149/2.1501713jes.

[21] Robinson JB, Darr JA, Eastwood DS, Hinds G, Lee PD, Shearing PR, et al. Non-uniform temperature distribution in Li-ion batteries during discharge - A combined thermal imaging, X-ray micro-tomography and electrochemical impedance approach. J Power Sources 2014. https://doi.org/10.1016/j.jpowsour.2013.11.059.

[22] Hausbrand R, Cherkashinin G, Ehrenberg H, Gröting M, Albe K, Hess C, et al. Fundamental degradation mechanisms of layered oxide Li-ion battery cathode materials: Methodology, insights and novel approaches. Mater Sci Eng B Solid-State Mater Adv Technol 2015. https://doi.org/10.1016/j.mseb.2014.11.014.

[23] Sharma N, Peterson VK, Elcombe MM, Avdeev M, Studer AJ, Blagojevic N, et al. Structural changes in a commercial lithium-ion battery during electrochemical cycling: An in situ neutron diffraction study. J Power Sources 2010. https://doi.org/10.1016/j.jpowsour.2010.06.114.

[24] Wang XL, An K, Cai L, Feng Z, Nagler SE, Daniel C, et al. Visualizing the chemistry and structure dynamics in lithium-ion batteries by in-situ neutron diffraction. Sci Rep 2012. https://doi.org/10.1038/srep00747. 
[25] Waag W, Käbitz S, Sauer DU. Experimental investigation of the lithium-ion battery impedance characteristic at various conditions and aging states and its influence on the application. Appl Energy 2013. https://doi.org/10.1016/j.apenergy.2012.09.030.

[26] Pastor-Fernández C, Uddin K, Chouchelamane GH, Widanage WD, Marco J. A Comparison between Electrochemical Impedance Spectroscopy and Incremental Capacity-Differential Voltage as Li-ion Diagnostic Techniques to Identify and Quantify the Effects of Degradation Modes within Battery Management Systems. J Power Sources 2017. https://doi.org/10.1016/j.jpowsour.2017.03.042.

[27] Schindler S, Danzer MA. A novel mechanistic modeling framework for analysis of electrode balancing and degradation modes in commercial lithium-ion cells. J Power Sources 2017. https://doi.org/10.1016/j.jpowsour.2017.01.026.

[28] Hsieh AG, Bhadra S, Hertzberg BJ, Gjeltema PJ, Goy A, Fleischer JW, et al. Electrochemicalacoustic time of flight: In operando correlation of physical dynamics with battery charge and health. Energy Environ Sci 2015. https://doi.org/10.1039/c5ee00111k.

[29] Robinson JB, Maier M, Compton T, Alster G, Brett DJL, Shearing PR. Spatially resolved ultrasound diagnostics of Li-ion battery electrodes. Phys Chem Chem Phys 2018. https://doi.org/10.1039/c8cp07098a.

[30] Robinson JB, Pham M, Kok MDR, Heenan TMM, Brett DJL, Shearing PR. Examining the Cycling Behaviour of Li-Ion Batteries Using Ultrasonic Time-of-Flight Measurements. J Power Sources 2019;444.

[31] Galushkin NE, Yazvinskaya NN, Galushkin DN. Mechanism of Gases Generation during Lithium-Ion Batteries Cycling. J Electrochem Soc 2019. https://doi.org/10.1149/2.0041906jes.

[32] Galushkin NE, Yazvinskaya NN, Galushkin DN. Mechanism of Thermal Runaway in Lithium-Ion Cells. J Electrochem Soc 2018. https://doi.org/10.1149/2.0611807jes.

[33] Davies G, Knehr KW, Van Tassell B, Hodson T, Biswas S, Hsieh AG, et al. State of Charge and State of Health Estimation Using Electrochemical Acoustic Time of Flight Analysis. J Electrochem Soc 2017. https://doi.org/10.1016/j.amjcard.2005.12.032.

[34] Gold L, Bach T, Virsik W, Schmitt A, Müller J, Staab TEM, et al. Probing lithium-ion batteries' state-of-charge using ultrasonic transmission - Concept and laboratory testing. J Power Sources 2017. https://doi.org/10.1016/j.jpowsour.2017.01.090.

[35] Ladpli P, Kopsaftopoulos F, Chang FK. Estimating state of charge and health of lithium-ion batteries with guided waves using built-in piezoelectric sensors/actuators. J Power Sources 2018. https://doi.org/10.1016/j.jpowsour.2018.02.056.

[36] Steingart DA. Nodeforwarder. GitHub 2018. https://github.com/dansteingart/nodeforwarder.

[37] Drakopoulos M, Connolley T, Reinhard C, Atwood R, Magdysyuk O, Vo N, et al. I12: The Joint Engineering, Environment and Processing (JEEP) beamline at Diamond Light Source. J Synchrotron Radiat 2015. https://doi.org/10.1107/S1600577515003513. 
[38] Ren D, Feng X, Lu L, Ouyang M, Zheng S, Li J, et al. An electrochemical-thermal coupled overcharge-to-thermal-runaway model for lithium ion battery. J Power Sources 2017. https://doi.org/10.1016/j.jpowsour.2017.08.035. 